\title{
Specific Contact Resistivity of Metal-Semiconductor Contacts-A New, Accurate Method Linked to Spreading Resistance
}

\author{
GARY P. CARVER, MEMBER, IEEE, JOSEPH J. KOPANSKI, MEMBER, IEEE, \\ DONALD B. NOVOTNY, MEMBER, IEEE, AND RICHARD A. FORMAN
}

\begin{abstract}
A new method to determine the specific contact resistivity of metal-semiconductor contacts has been developed. It allows the separation of the total series resistance between two contacts into the contributing component resistances. The principle of the method is the subtraction of the semiconductor spreading resistance from the total two-contact resistance. This requires geometrically well-defined small contacts that are fabricated precisely by lithographic methods. Using this method, accurate values were obtained for the specific contact resistivity of an aluminum-1.5-percent silicon alloy to p-type silicon wafers having dopant densities from $5 \times 10^{14}$ to $2 \times 10^{20} \mathrm{~cm}^{-3}$. The specific contact resistivity values are lower than previously published values obtained using earlier methods in which parasitic and nonideal effects could not be quantified or eliminated. The lower values indicate that contact resistance has a less limiting effect on the performance of integrated circuits than presently believed.
\end{abstract}

Key Words-Contact resistance, contact resistivity, contacts, integrated circuits, metal-semiconductor contacts, Schottky barriers, Schottky diodes, silicon, specific contact resistivity, spreading resistance.

\section{INTRODUCTION}

$\mathrm{T}$ HE metal-to-semiconductor contact is the oldest and most common device structure used in integrated circuits, and it may be the most poorly characterized. The accurate quantification of contact performance is inhibited by a lack of knowledge on a microscopic scale of the interface and nearby bulk material, and by difficulties in accurately measuring the interface electrical characteristics. Many of the published measurements on contact resistance are flawed by the incomplete separation of interface and bulk material properties, the inability to measure accurately the desired parameters, the use of models that are too simple, or an improper or nonstandard application of terminology [1], [2]. The metal-to-semiconductor contact resistance is not easily modeled because of complex material- and process-dependent effects that occur at the interface. Therefore, actual measurement of the contact resistance is required.

Existing methods and structures, especially two-level planar test devices, incorporate parasitic resistances

Manuscript received April 27, 1987; revised November 16, 1987 The authors are with the Semiconductor Electronics Division, National Bureau of Standards, Gaithersburg, MD 20899.

IEEE Log Number 8718962. caused by nonuniform current flow. These parasitic resistances interfere with the evaluation of the specific contact resistivity, the parameter that characterizes the contact resistance of a uniform contact.

We have developed a new resistance measurement method that allows accurate determination of the metalto-semiconductor specific contact resistivity. The method can be used to measure metal-to-semiconductor interfacial resistance of semiconductor samples with uniform bulk resistivity or samples having a nonuniform resistivity depth profile, including layered structures.

Our method requires two or more small contacts; the number of contacts needed is dictated by the three-dimensional resistivity profile of the sample and the certainty with which that profile is known. The key element of the measurement is the subtraction of the spreading resistance at two lithographically fabricated, geometrically well-defined contacts from the total resistance measured by probing the two contacts. In the case of a uniform-resistivity semiconductor substrate, the spreading resistance is calculated from the resistivity obtained from a four-contact measurement made with the same contacts.

Using this method, we measured the specific contact resistivity between an aluminum-1.5-percent silicon alloy and boron-doped bulk silicon samples having a bulk dopant density over the range from $5 \times 10^{14}$ to $2 \times 10^{20}$ $\mathrm{cm}^{-3}$.

The values of specific contact resistivity that we obtain are lower than values reported by others who used conventional contact resistance methods. Our results confirm the conclusion of a recent analysis [3], [4]; conventional models used with existing two-level planar contact resistor structures overestimate the contact resistance. Our results show that contact resistance will limit the performance of integrated circuits less than is presently believed.

\section{Definitions and a Perspective on Contact Resistance Measurements}

To avoid confusion over terminology, it is important to define the words used here. The electrical resistance of a contact between two different materials must depend on the total current through the contact and the two equipo- 
tential planes between which the voltage is measured. The measured "contact resistance" is not a useful predictive parameter unless the current distribution is known in detail and the geometry of the current and potential distributions are applicable to other contacts. Generally, the value of the "contact resistance" is dependent upon the particular structure and measurement and includes material- and geometry-dependent parameters, such as the sheet resistance of the conducting layers and the contact area.

The only measurement-method-independent parameter that describes the resistance of the interface at the contact is called in this paper the "specific contact resistivity," and is given in units of ohms times square centimeters. This term is the one most often used in the literature to parameterize the interface; however, it is not always given the same meaning and is not always method independent. In this paper, the "specific contact resistivity" is defined, based on the assumptions and mathematical expressions to follow, as the resistance of a unit area of the thin interfacial layer between the bulk metal and semiconductor substrate.

Other authors have used a variety of other terms for the same quantity. For example, it is called the "specific interface resistance" in [2], the "specific contact resistance" in [5]-[7], the "contact resistivity" in [8], and the "specific resistance" in [9]. In still other cases, the same term as used here, "specific contact resistivity," in the same units, has been used to represent a quantity that is not independent of the structure used to make the measurement because it includes properties of the materials near the interfacial layer or includes parasitic contributions. One well-known example can be found in [10].

One theoretical definition of the specific contact resistivity $\rho_{C}$ is

$$
\rho_{C}=\lim _{J \rightarrow 0} \partial V / \partial J
$$

The symbol $J$ represents the current density and $V$ indicates the voltage across the interfacial layer.

The implicit assumption underlying (1) is that $J$ is uniform across the contact interface. But $J$ is not a measurable parameter; what is measured is the value of the current. Therefore, it may be better to require that the current density is kept constant as the area of the contact approaches zero in the limit, as was done in [10]. The application of this condition for our method is discussed in the next section.

The current-voltage behavior of the contact used experimentally to deduce $\rho_{C}$ has great practical importance. Real contacts carry nonzero currents and have nonzero voltage drops across their interface. Unless they behave linearly, and symmetrically with respect to the current direction, that is, "ohmically," they may not be useful. Generally, therefore, (1) is applied to contacts that, over a specified range of current, are ohmic. This aspect of contact behavior is discussed extensively elsewhere [1], [7], [9].
Since the specific contact resistivity cannot be measured directly but must be inferred from a measurement on a real contact, some authors have sought to differentiate the measured quantity, the deduced quantity that includes parasitic and nonideal effects, and the conceptually ideal quantity (one that does not include parasitic and nonideal effects). An example is the use of the terms "contact resistance," "'specific interface resistance," and "specific contact resistance" in [2].

The measurement method used in this work includes a rigorous formulation of the measured quantities in terms of clearly defined parameters. The measured quantity is the total resistance between two contacts and the deduced quantity that describes the electrical behavior of the interface is the specific contact resistivity. Where the actual parameters, such as the current distribution across the interface, cannot be obtained exactly they are bounded by appropriate limiting cases.

The benefits, drawbacks, and limitations of previously used contact resistance structures and methods have been discussed in detail by Cohen [2]. The methods may be categorized into two-, three- and four-terminal measurements. The most commonly used methods in the recent literature are the transmission line tap resistor method [10], [11], a three-terminal method, and the four-terminal cross-bridge Kelvin contact resistor method [5], [12], [13]. Since these are modeled as two-level structures composed of uniform planar layers, the results are sensitive to the actual surface dopant concentration of the semiconductor layer beneath the contact, the current uniformity in the vertical direction [3], [4], the level-to-level alignment of the structure [14], and other parasitic effects [15]. The goal of the analyses and calculations that have been developed here is to more accurately separate the parasitic and nonideal effects from the measured resistance in such two-level structures so as to more accurately determine the specific contact resistivity [4], [11], [16][18].

Two-terminal methods for measuring contact resistance would seem to be simpler conceptionally and to have a greater likelihood of avoiding the disadvantages of methods that have more than the minimum necessary two terminals. The intuitively simplest such structure, only marginally suitable for today's integrated circuit materials, is the solid sample with a uniform cross section and contacts on the ends [19].

All two-contact structures possess the inherent difficulty that the interface resistance is in series with the bulk resistance of the structure. To separate the interface and bulk resistance, the two-contact structure is usually multiply replicated, with each replication having different dimensions. Because the components of the total resistance between the two contacts are in series, they cannot be measured or determined independently; instead, their values are inferred by their dependence on another parameter or by the way they scale with the varied dimensional parameter. In the method of Reeves and Harrison [20], for example, the inferred parameter is the contact attenuation 
constant appropriate to the transmission-line model [21]. On the other hand, the method of Cox and Strack [6] utilizes several round contacts of different diameters and one large backside contact. The resistance is measured between each top contact and the one backside contact. Curve-fitting techniques are then used to separate the linear dependence of the contact resistance on the contact area from the nonlinear spreading resistance dependence on area, and from the constant backside-contact resistance.

Our method is related to the method of Cox and Strack [6] in that the spreading resistance and other noninterface components of the resistance between two contacts are separated from the total to determine the contact resistance component. The difference is that, in our method, the contacts are configured to allow the direct calculation of the spreading resistance and, because two equivalent contacts are used, there is no backside resistance to be determined. The spreading resistance term is independently evaluated and no curve fitting is required. The specific contact resistivity is directly determined within uncertainty limits that can be quantified. In this paper, application of the method to uniform resistivity samples is aided by the agreement in the values of the substrate resistivity as determined from four-contact resistivity measurements and from spreading resistance measurements made on geometrically well-defined contacts. We have previously demonstrated this agreement [22].

\section{Measurement of the Contact Resistance at Geometrically Well-Defined Small Contacts}

The essence of this measurement is that the specific contact resistivity is determined by measuring the total resistance at a contact and then subtracting each of the contributing series resistances until only the contact resistance component remains. The specific contact resistivity $\rho_{C}$ is then determined by multiplying the contact resistance by the area of the contact.

Our measurements were performed on commercial silicon wafers with diameters of 2 or 3 in, using small contacts that are geometrically defined by a square array of etched windows in a grown silicon dioxide layer. Each round window is within a square metallized area that serves as a probe pad, as shown in Fig. 1(a). A cross section through one such contact is shown in Fig. 1(b). Micropositioner probes are used to contact the aluminum1.5-percent silicon alloy probe pads.

To carry out the measurements, the area and geometry of the two similar contacts, the spreading resistance in the semiconductor, and the probe and probe pad resistance must be known. Assuming the resistances are ohmic over the range of the measuring current, the specific contact resistivity is given by

$$
\rho_{C}=1 / 2\left\{A\left[R_{T}-R_{P}-R_{S P}\right]\right\} .
$$

Here, $R_{T}$ is the total measured resistance between the two contacts, $R_{P}$ is the probe and probe pad resistance for the

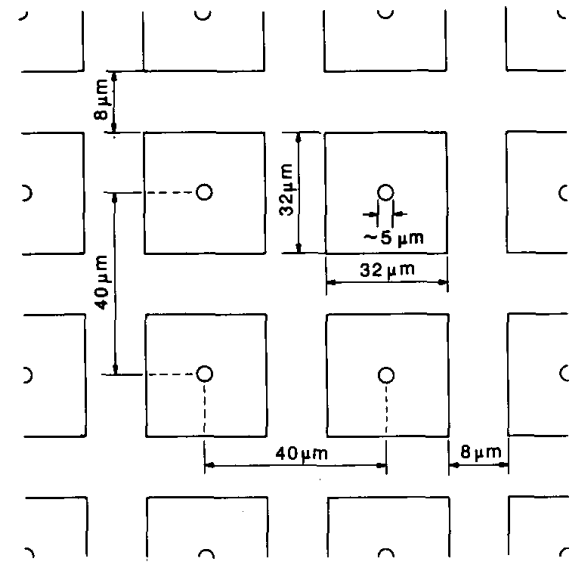

(a)

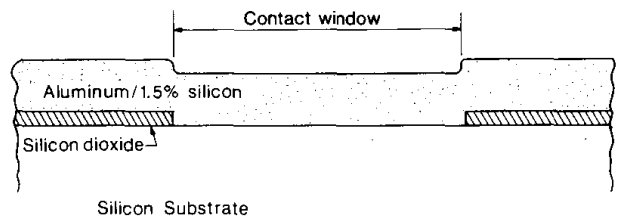

(b)

Fig. 1. The contact and probe pad array. (a) Layout and dimensions of the approximately $5-\mu \mathrm{m}$-diameter contact windows and the $32-\mu \mathrm{m}$ by $32-\mu \mathrm{m}$ probe pads. (b) A cross section of one contact window.

two contacts, including the spreading resistance in the probe and probe pad, and $R_{S P}$ is the spreading resistance for the two contacts. $A$, as before, denotes the contact area of one of the two similar contacts.

The spreading resistance $R_{S P}$ for two flat nonindenting circular contacts of radius $a$ on the surface of an $n$-layered structure may be written [23]

$$
R_{S P}=\left(\rho_{n} / 2 a\right) C_{n}
$$

where $\rho_{n}$ is the resistivity of the $n$th layer and $C_{n}$ is a correction factor that depends on the resistivities and thicknesses of the layers in the multilayer structure, on the contact diameter and separation, and on the current distribution in the contacts. For widely separated contacts to a uniform semi-infinite medium, $\rho_{n}$ is the bulk resistivity of the medium and $C_{n}$ is unity [24].

The area $A$ is determined by measuring the dimensions of the contact. We measured the contact dimensions using an optical image-shearing micrometer eyepiece on a microscope. Because our contacts are planar, we used the lateral dimensions of the contact window in the oxide to calculate the actual contact area. This represents the single largest source of uncertainty in our present results; the uncertainty could be reduced by measuring the contact window diameter more accurately.

The total resistance $R_{T}$ is measured between two contacts in series. To verify that each single contact behaves ohmically, measurements were made between one contact probe pad and the backside of the wafer. 
The total resistance was measured using a constant current source and digital voltmeter. The resistance is the slope of the current versus voltage data. For all results reported here, the slope was constant within 3 percent for current values covering a range of about a factor of five. The current typically was set between 0.1 and $100 \mu \mathrm{A}$, depending on the substrate resistivity, and the power dissipated was less than about $0.6 \mu \mathrm{W}$. This power is much less than the power observed to cause a measurable change in the resistance due to heating.

The probe and probe pad resistance is the combined resistance in the metal probes used to make contact to the probe pads and the resistance in the aluminum probe pads. This resistance is measured by measuring the resistance between two probes when both contact the same aluminum pad. The value of the probe and probe pad resistance is about $0.5 \Omega$ per probe and is smaller than all other series resistance components.

The bulk resistivity of our uniformly doped substrates was measured by the in-line four-contact method [25]. Typically, four measurements were made using 16 contacts of the contacts in the center region of the wafer. The measurements were averaged and corrected for the thickness of the wafer using an expression appropriate to an insulating backside [26]. The same procedure for the fourcontact resistivity can be applied to the case of a substrate with a nonuniform vertical resistivity profile, by the use of the appropriate correction factors [26].

\section{Uncertainties in the SPREAding Resistance and the Specific Contact Resistivity}

The expression (see (2)) for the specific contact resistivity includes the assumption that the current density across the interfacial layer is uniform. The correction factor in the spreading resistance depends upon the current distribution in the contact. However, the current distribution is determined both by the series resistance at the contact and by the resistivity-depth profile in the semiconductor.

In the case of a pure spreading resistance contact of the geometry already described to a uniform resistivity substrate, the current distribution through the contact must have a current density of the form discussed by Schumann and Gardner [27], [28]

$$
J(r)=1 /\left[2 \pi a\left(a^{2}-r^{2}\right)^{1 / 2}\right]
$$

where $r$ is the distance from the center of the round contact and $J(r)$ is zero for $r>a$.

The actual current distribution in our samples is intermediate between the uniform and Schumann-Gardner distributions. In the limit when the contact resistance is very large, the current tends toward a uniform distribution. This can be seen by considering the spreading resistance for a conducting layer on a much more highly conducting layer, which approximates a uniform current flow through the contact [23]. For our samples, the interfacial layer is analogous to a thin layer that has a lower conductivity than the thick sample beneath. In the other limit, when the contact resistance is very small compared to the spreading resistance, the current tends toward the Schumann-Gardner current distribution [23]. In this case, the current flow is controlled entirely by the (pure) bulk spreading resistance in the semiconductor.

Ideally, the complete situation, the planar contact having contact resistance that is in series with the spreading resistance on one side of the contact, should be calculated self-consistently to obtain the correct current distribution and specific contact resistivity. This approach can be adapted to actual layered structures, such as a planar contact to a junction-isolated conducting implanted layer. However, that calculation is not necessary for the samples measured in this study.

Our approach was to calculate the spreading resistance, using the results of the four-contact resistivity measurement, for the two different model current distributions appropriate for a uniform resistivity substrate, including the resistance between the two contacts. We then deduced the value of the specific contact resistivity separately for each case. The calculations were carried out using the trapezoidal Rhomberg integration technique to evaluate the correction factor integrals for the two current distributions [23]. For all samples in this study, the correction factor for the Schumann-Gardner current distribution was 0.988 and for the uniform current distribution it was 1.07 . The effect of the correction factors are to raise the specific contact resistivity by 1 to 10 percent (Schumann-Gardner) and to lower it by 5 to 36 percent (uniform), depending upon the thickness of the sample.

For our results, all remaining measurement uncertainty in the determined value of the contact resistivity arises from the uncertainties that were described earlier in the measurement of the contact geometry and in the voltage and current measurements. Assuming, initially, that nonuniformities in material and geometric parameters are small, then the largest uncertainty in the determination of the contact resistance derives from the measurement of the diameter of the contact windows. This is not simply because the contact window measurement is less accurate, but also because the specific contact resistivity depends upon the square of the contact radius. In terms of the measured parameters, the specific contact resistivity for one round planar contact is

$$
\rho_{C}=\left(\pi a^{2} V / I\right)-\pi^{2} a(s / 2)\left(V_{4} / I_{4}\right)
$$

where $V$ and $I$ are the voltage and current measured through the contact, $V_{4}$ and $I_{4}$ are the voltage and current for the four-contact measurement, and $s$ is the contact separation in the four-contact array. (The probe resistance and the four-contact resistivity correction factors are neglected in this expression.) Assuming that the random errors in the measurements of the five quantities are uncorrelated, a simple propagation of error analysis shows that the variance in $\rho_{C},\left(\delta \rho_{C}\right)^{2}$, is at least four times as great 
as the variance in $a$

$$
\begin{aligned}
\left(\frac{\delta \rho_{C}}{\rho_{C}}\right)^{2}= & {\left[1+\frac{\pi a}{8} \frac{\rho}{\rho_{C}}\right]^{2}\left(\frac{2 \delta a}{a}\right)^{2} } \\
& +\left[1+\frac{\pi a}{4} \frac{\rho}{\rho_{C}}\right]^{2}\left[\left(\frac{\delta V}{V}\right)^{2}+\left(\frac{\delta I}{I}\right)^{2}\right] \\
& +\left[\frac{\pi a}{4} \frac{\rho}{\rho_{C}}\right]^{2}\left[\left(\frac{\delta V_{4}}{V_{4}}\right)^{2}+\left(\frac{\delta I_{4}}{I_{4}}\right)^{2}\right]
\end{aligned}
$$

The uncertainty contributions proportional to $(a \rho) / \rho_{C}$ indicate that the smaller the contact radius or the smaller the bulk resistivity, the more accurate the measurement of the specific contact resistivity.

These conclusions may be understood by considering that the contact resistance is obtained by subtracting the resistance caused by the probes and the resistance due to the semiconductor substrate from the total resistance at the contact. As a general rule, the larger the contact resistance in relation to the other resistances, the more accurately it may be measured.

Since, for our samples, the relation between the specific contact resistivity and the bulk resistivity is fixed by their dependence upon the dopant density in the silicon substrate, and since the probe resistance is constant, the only variable parameter is the contact radius. Thus, the smaller the contact radius, the larger the contact resistance in comparison to the other resistances. For the case of thick uniform-resistivity substrates; as the contact radius is decreased, the contact resistance increases as the inverse square of the radius while the bulk resistance increases only in proportion to the inverse first power of the radius. In the limit of vanishingly small $A$, the contact resistance due to the interfacial layer approaches 100 percent of the total measured resistance. (This is generally true, even for samples with nonuniform resistivity-depth profiles.)

The dominance of the contact resistance for small-area contacts may be seen directly by rewriting (4) in terms of the contact area and the contact resistance measuring current density $J$

$$
\rho_{C}=\frac{V}{J}-A^{1 / 2} \pi^{3 / 2} \frac{s}{2} \frac{V_{4}}{I_{4}}
$$

In the limit that the contact area is reduced while the current density is held constant, the specific contact resistivity is just the first term in (7). This result is consistent with the definition of specific contact resistivity used by Berger [10] and may be formulated

$$
\rho_{C}=\lim _{A \rightarrow 0}(\partial V / \partial J) .
$$

The constant probe resistance became a significant factor only for our very high dopant density samples. For samples with a dopant density of $10^{15} \mathrm{~cm}^{-3}$ and contact radii about $2.5 \mu \mathrm{m}$, the total resistance at each contact is about $1.8 \times 10^{4} \mathrm{\Omega}$; of this the probe resistance is about $0.5 \Omega$. For this sample, the contact resistance is about 4.4 $\times 10^{3} \Omega$ and the spreading resistance in the silicon is about
$1.3 \times 10^{4} \Omega$. For samples with a higher dopant density of $1 \times 10^{20} \mathrm{~cm}^{-3}$, the $0.5-\Omega$ probe resistance becomes about 10 percent of the total resistance.

Nonuniformities in the contact radius or in the lateral resistivity of the silicon substrate can become large enough to affect the accuracy of the measurement. In our processing, variations in the contact window radii were too small to contribute measurably to the uncertainty. When measurements are made on substrates known to have a very uniform resistivity, such as wafers with epilayers, the variation in measured resistance at the contacts is less than 1 percent. This is much smaller than the estimated uncertainty in the average measured value of the contact radius. Typical commercial $\mathrm{CZ}$ wafers, however, have resistivity variations as large as 10 percent or more [29]. To mitigate the effect of resistivity variations on the calculations, the specific contact resistivity results from 8 to 16 two-contact resistance measurements and 4 four-contact resistance measurements in the center region of the wafers were averaged.

\section{Contact-Resistivity Sample Preparation}

The samples in this study are silicon wafers having diameters of 50 or $75 \mathrm{~mm}$. The aluminum-to-silicon contact takes place at contact windows etched through thermally grown silicon dioxide. The contacts are in a square array with a $40-\mu \mathrm{m}$ center-to-center spacing. The aluminum is patterned into square probe pads $32 \mu \mathrm{m}$ on a side. For the data reported in this paper, the contact window radius was about $2.4 \mu \mathrm{m}$.

The reproducibility and accuracy of the results derive from the controlled formation of aluminum-to-silicon contacts having well-defined electrical and geometrical characteristics. To form such contacts, the following procedures were performed. The wafers were cleaned and then oxidized (in an atmosphere of dry oxygen at a temperature of $1050^{\circ} \mathrm{C}$ ) to form an oxide thickness of approximately $200 \mathrm{~nm}$. The contact windows were patterned in photoresist and etched in a buffered hydrofluoric acid solution. The wafers were metallized by vacuum sputtering aluminum with 1.5 -percent silicon to a thickness of about $700 \mathrm{~nm}$. The photoresist was patterned and the aluminum was etched to form the contact pads. The wafers were then sintered at $450^{\circ} \mathrm{C}$ in a 10 -percent forming gas atmosphere for $20 \mathrm{~min}$.

\section{The Specific Contact Resistivity as a} Function of The Dopant Density of THE Silicon

Values of the specific contact resistivity determined using our new method are shown in Fig. 2. There, the specific contact resistivity is plotted as a function of the four-contact bulk resistivity. The spreading resistance correction factor was set equal to one. The silicon resistivity was measured using contacts from among the contacts that were used for the contact resistance measurements. 


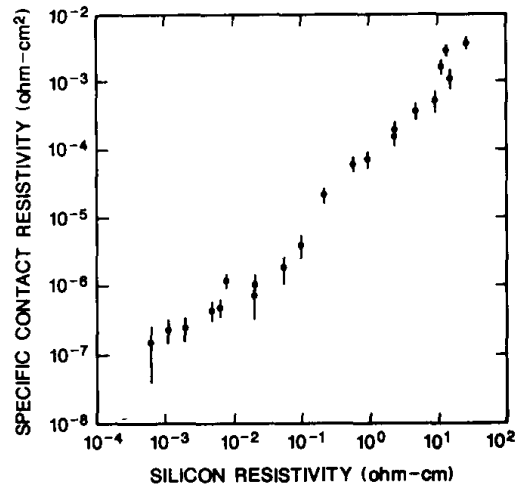

Fig. 2. The aluminum-to-silicon specific contact resistivity as a function of the silicon bulk resistivity. The bulk resistivity was measured using the in-line four-contact method.

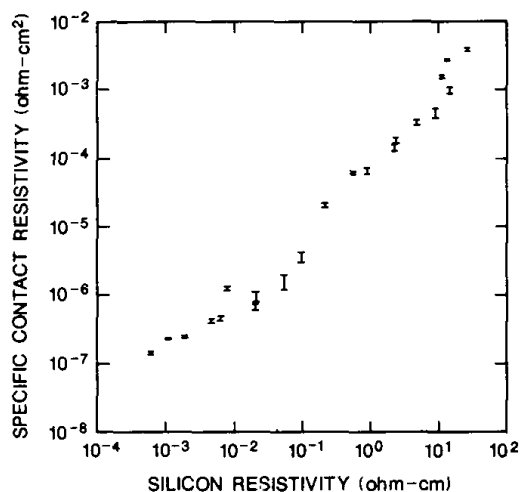

Fig. 3. The specific contact resistivity data of Fig. 2 adjusted by calculating the spreading resistance component using a uniform current density through the contact (lower limit) and using a Schumann-Gardner current density through the contact (upper limit).

The effects of the correction factors for the two limiting forms of the current density are shown in Fig. 3.

Measurements made on samples with contact window radii of about $5.1 \mu \mathrm{m}$, about twice as large as those used for the data presented here, yielded values for the specific contact resistivity that were the same as the values of the smaller windows, to well within estimated uncertainties. This agreement indicates that the interfacial contact region is uniform on a scale below the size of our contacts. Because the measured resistance of the interfacial layer is directly proportional to the contact area, we can conclude that the deduced specific contact resistivity has a unique value for our contacts.

In Fig. 4, the data are plotted against the dopant density [30] and are compared to predictions of the thermionic field emission model [31]. In previous work, specific contact resistivity data were found to agree with this model when the value of the tunneling effective mass is about 0.5 and the barrier height is in the range 0.6-0.7 eV [8], [31]. It can be seen that our data do not follow a curve of constant barrier height. We lack theoretical understanding of this behavior; this feature is still under study.

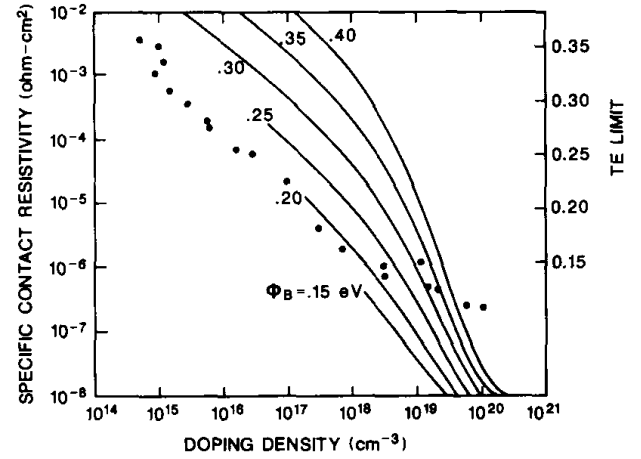

Fig. 4. The specific contact resistivity as a function of doping concentration and barrier height calculated using the equations in [33]. In this model, $\rho_{c}$ is predicted in three regions: the field emission region (FE) for high doping concentrations, the thermionic emission limit (TE) at low doping, and the thermionic-field emission region (TFE) between the two limits. The solid lines are the results of the calculation in the TFE region. The values of the TE limits in electron volts are shown on the right-side axis. The calculation was performed using a tunneling effective mass of $m^{*} / m_{0}=0.66$, at a temperature of $300 \mathrm{~K}$.

\section{Comparison of the New Contact Resistivity Data with Currently Quoted Values and with EFfects of Contact Resistance on Circuits}

The results presented here are accurately measured values of the specific contact resistivity for aluminum on boron-doped silicon. Values of the specific contact resistivity, when the contacts are fabricated as described in this paper, are significantly smaller than those previously reported by many researchers using different measurement methods. It is apparent that for each different method, including the one used here, the resistance of the interfacial layer between the metal and semiconductor is linked with the resistance of the metal and semiconductor regions on either side of the interface. The current distribution through the contact is determined by the composite structure. Even if it were possible to separate the interface resistance, the accepted definition of specific contact resistivity could not be applied because it requires uniform current density. These and other practical constraints have caused previously measured values to exhibit large scatter and to be too large.

How are these considerations important in real circuits? In actual circuit contacts there is current crowding, nonuniform current density, and other parasitic and nonideal effects that contribute to the total resistance at the contact. It is the total resistance that influences the performance of that portion of the circuit, such as an interconnect. Therefore, it would seem that realistic contact configurations should be measured to obtain the contact resistance. But this may not yield results that can be used to predict the performance of other contact configurations.

We believe it is far better to know accurately the individual parameters that contribute to a composite effect and to model the various real structures to predict their performance. The front and end contact resistances [12], [32] can be calculated for a variety of contact structures without actually fabricating the structures. A circuit designer 
could then adjust the local surface dopant concentration or the implant dose to meet the needs of the device when the specific contact resistivity and contact geometry are known [8], [33]. Knowing the value of the specific contact resistivity for the materials of interest provides the opportunity to know the performance of a contact structure in advance.

Another measurement-related aspect to contact resistance measurements is that even with an ideal geometrical configuration, only the product of the specific contact resistivity and the contact area can be measured electrically. At least one separate optical measurement of length is required in all methods to determine the specific contact resistivity. This means that electrical measurements can be used to monitor at the same time the contact area and the contact metallurgy. Variations in the value of each separately can be obtained by measuring optically the contact area and comparing the calculated specific contact resistivity with the expected results. Alternatively, since the aluminum-silicon contact can be formed with uniform and reproducible properties [34], the value of the specific contact resistivity can be used to monitor the contact area and the silicon dopant concentration at the interface.

Because we have shown that the specific contact resistivity between aluminum and silicon is smaller than previously reported, we conclude that contact resistance in integrated circuits does not contribute as much in propagation time delay as presently believed [35]. The predicted usefulness of aluminum for contact dimensions no smaller than about $1.0 \mu \mathrm{m}$ can be revised downward; our results, incorporated into at least one model [36], indicate that in a CMOS process, aluminum metallurgy will be useful for contact dimensions as small as about $0.5 \mu \mathrm{m}$.

\section{ApPlication to NonUniform Resistivity-Depth Profiles}

In the cases of samples having a nonuniform resistivitydepth profile and samples having a conducting layer with a thickness that is not very much larger than the diameter of the contacts, the method presented in this paper and demonstrated on uniform resistivity samples still provides accurate results. Even with such samples, the method requires less processing, fewer measurements, and less area on the sample than other contact resistance methods and structures.

\section{A. When the Resistivity Profile is Known}

The procedure is to use (2). The total resistance between two contacts, the probe resistance, and the diameter of the contacts are measured. The spreading resistance is calculated.

For the uniform and thick samples reported in this paper, we first set the correction factor for the spreading resistance (see (3)) to one. Then to account for the effects of the contact resistance on the current distribution, correction factors appropriate to the Schumann and Gardner current distribution [27], [28] and to the uniform current distribution were calculated to show, in Fig. 3, the limiting values of the specific contact resistivity.

For samples with nonuniform resistivity-depth profiles, or for very thin samples, the spreading resistance between two contacts is calculated using the formulation in [23], which gives the correction factors for the two-contact spreading resistance on the top surface of a layered resistivity structure [23, eqs. 15-17].

\section{B. When the Resistivity Profile Is Not Known}

The separation of the contact resistance and the spreading resistance is accomplished experimentally. The most straightforward procedure is to measure the total resistance between two pairs of contacts, both to the desired resistivity profile but one pair having the surface of the semiconductor ion implanted to increase the surface dopant density. The difference between the pair of resistance measurements is twice the contact resistance at one (nonimplanted) contact.

Even where the surface dopant density of the target profile is already high, a shallow ion implantation can significantly reduce the contact resistance. The amount of reduction and therefore the residual error in the result can be estimated by examination of the specific contact resistivity versus dopant density curves for the applicable system.

\section{ACKNOWLEDGMENT}

The authors greatfully acknowledge the assistance of $\mathrm{J}$. Albers and J. Ehrstein in this work. Our achievement was enhanced by their support.

The authors also thank the staff of the NBS Semiconductor Processing Research Laboratory for their assistance in the careful preparation of the samples.

\section{REFERENCES}

[1] H. K. Henish, Semiconductor Contacts, An Approach to Ideas and Models. Oxford: Clarendon, 1984, ch. 1.

[2] S. S. Cohen, "Contact resistance and methods for its determination," Thin Solid Films, vol. 104, pp. 361-379, 1983.

[3] K. C. Saraswat, W. M. Loh, T. A. Schreyer, and S. E. Swirhun, "Measurement and extraction of specific contact resistivity," in Proc. 3rd Int. IEEE VLSI Multilevel Interconnection Conf. (Santa Clara, CA), pp. 385-391, June 1986.

[4] W. M. Loh, S. E. Swirhun, T. A. Schreyer, R. M. Swanson, and K. C. Saraswat, "Modeling and measurement of contact resistances," IEEE Trans. Electron Devices, vol. ED-34, no. 3, pp. 512-524, Mar. 1987.

[5] S. J. Proctor and L. W. Linholm, "A direct measurement of interfacial contact resistance," IEEE Electron Device Lett., vol. EDL-3, no. 10 , pp. 294-296, Oct. 1982

[6] R. H. CoX and H. Strack, "Ohmic contacts for GaAs devices," SolidState Electron., vol. 10, pp. 1213-1218, 1967.

[7] S. M. Sze, Physics of Semiconductor Devices, 2nd ed. New York: Wiley, 1981, p. 304.

[8] D. K. Schroder and D. L. Meier, "Solar cell contact resistance-A review," IEEE Trans. Electron Devices, vol. ED-31, no. 5, pp. 637647, May 1984

[9] E. H. Rhoderick, "Metal semiconductor contacts,' IEE Proc., vol. 129 , no. 1 , pp. 1-14, 1982.

[10] H. H. Berger, "Contact resistance and contact resistivity," J. Electrochem. Soc., vol. 119, no. 4, pp. 507-514, Apr. 1972.

[11] G. K. Reeves and H. B. Harrison, "Obtaining the specific contact resistance from transmission line model measurements," IEEE Electron Device Lett., vol. EDL-3, no. 5, pp. 111-113, May 1982. 
[12] S. J. Proctor, L. W. Linholm, and J. A. Mazer, "Direct measurements of interfacial contact resistance, end contact resistance, and interfacial contact layer uniformity,' IEEE Trans. Electron Devices, vol. ED-30, no. 11, pp. 1535-1542, Nov. 1983.

[13] J. Chern and W. G. Oldham, "Determining specific contact resistivity from contact end resistance measurements," IEEE Electron Device Lett., vol. EDL-5, pp. 178-180, May 1984.

[14] R. L. Gillenwater, M. J. Hafich, and G. Y. Robinson, "Extraction of minimum specific contact resistivity using Kelvin resistors,' IEEE Electron Device Lett., vol. EDL-12, pp. 674-676, Dec. 1986.

[15] A. A. Naem and D. A. Smith, "Accuracy of the four-terminal measurement techniques for determining contact resistance," J. Electrochem. Soc., vol. 133, no. 11, pp. 2377-2380, Nov. 1986.

[16] J. A. Mazer, L. W. Linholm, and A. N. Saxena, "An improved test structure and Kelvin measurement method for the determination of integrated circuit front contact resistance," J. Electrochem. Soc., vol. 132 , no. 2 , pp. $440-443,1985$.

[17] A. Scorzoni, M. Finetti, K. Grahn, I. Suni, and P. Cappelletti, "Cur rent crowding and misalignment effects as sources of error in contact resistivity measurements-Part I: Computer simulation of conventional CER and CKR structures," IEEE Trans. Electron Devices, vol ED-34, no. 3, pp. 525-532, Mar. 1987.

[18] M. Finetti, S. Guerri, P. Negrini, A. Scorzoni, and I. Suni, "Contact resistivity of silicon/silicide structures formed by thin film reactions," Thin Solid Films, vol. 130, pp. 37-45, 1985.

[19] W. M. Loh, P. J. Wright, T. A. Schreyer, S. E. Swirhun, K. C. Saraswat, and J. D. Meindl, "The sidewall resistor-A novel test structure to reliably extract specific contact resistivity," IEEE Elec tron Device Lett., vol. EDL-7, no. 8, pp. 477-479, 1986.

[20] G. Reeves and H. B. Harrison, "Determination of contact parameters of interconnecting layers in VLSI circuits," IEEE Trans. Electron Devices, vol. ED-33, no. 3, pp. 328-334, Mar. 1986.

[21] H. H. Berger, "Models for contacts to planar devices," Solid-State Electron., vol. 15, pp. 145-158, 1972.

[22] G. P. Carver, S. S. Kang, J. R. Ehrstein, and D. B. Novotny, "'Welldefined contacts produce accurate spreading resistance measurements,"' J. Electrochem. Soc., vol. 134, no. 11, pp. 2878-2882, 1987.

[23] J. Albers, "Some aspects of spreading resistance profile analysis," in Emerging Semiconductor Technology, ASTM STP 960, D. C. Gupta and P. H. Langer, Eds., Amer. Soc. for Testing and Materials, 1986, pp. 480-501.

[24] R. Holm, Electric Contacts; Theory and Application. New York: Springer-Verlag, 1967, p. 16.

[25] Standard Method for Measuring Resistivity of Silicon Slices with a Collinear Four-Probe Array, Designation: F84-84, Amer. Soc. for Testing and Materials, Philadelphia, PA.

[26] J. Albers and H. L. Berkowitz, "An alternative approach to the cal culation of four-probe resistances on nonuniform structures, "J. Electrochem. Soc., vol. 132, no. 10, pp. 2453-2456, 1985.

[27] P. A. Schumann and E. E. Gardner, "Application of multilayer potential distribution to spreading resistance correction factors," $J$. Electrochem. Soc., vol. 116, pp. 87-91, 1969.

[28] E. E. Gardner and P. A. Schumann, "Spreading resistance correction factors,"' Solid-State Electron., vol. 12, pp. 371-375, 1969.

[29] K. Senda, Y. Hiroshima, S. Matsumoto, T. Kuriyama, M. Susa, S Terakawa, and T. Kunii, "Fixed pattern noise in the solid-state imagers due to the striations in Czochralski silicon crystals," J. Appl. Phys., vol. 57, pp. 1369-1372, 1985.

[30] W. R. Thurber, R. L. Mattis, Y. M. Liu, and J. J. Filliben, "Resis tivity-dopant density relationship for boron-doped silicon," J. Electrochem. Soc., vol. 127, pp. 2291-2294, 1980.

[31] A. Y. C. Yu, "Electron tunneling and contact resistance of metalsilicon contact barriers,"' Solid-State Electron., vol. 13, pp. 239-247, 1970 .

[32] H. H. Berger, "Models for contacts to planar devices," Solid-State Electron., vol. 15, pp. 145-158, 1972.

[33] R. L. Maddox, "On the optimization of VLSI contacts,' IEEE Trans. Electron Devices, vol. ED-32, pp. 682-690, Mar. 1985.

[34] W. H. Class and J. F. Smith, in VLSI Handbook, N. G. Einspruch, Ed. Orlando, FL: Academic, 1985, p. 435

[35] H. B. Bakoglu and J. D. Meindl, "Optimal interconnection circuits for VLSI," IEEE Trans. Electron Devices, vol. ED-32, pp. 903-909, May 1985.

[36] D. M. Brown, M. Ghezzo, and J. M. Pimbley, “Trends in advanced process technology-Submicrometer CMOS device design and process requirements," Proc. IEEE, vol. 74, pp. I678-1702, Dec. 1986.

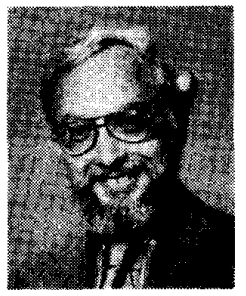

Gary P. Carver (M'79) received the B.S. degree in physics from Clarkson University, Potsdam, $\mathrm{NY}$, in 1963 and the Ph.D. degree from Cornell University, Ithaca, NY, in 1970 . His dissertation was on nuclear magnetic resonance characterization of graphite intercalation compounds.

After graduation, he began work in the Solid State Branch of the Naval Surface Weapons Center, White Oak. He became a principal investigator on research projects in transport properties of semiconductors. He designed a unique appa-

ratus and measured the ac Hall mobility in amorphous semiconductors. His research also included measuring the velocity-electric field relation using nanosecond pulse techniques and the magnetophonon resonance spectrum in $\mathrm{PbTe}$ and $\mathrm{PbSnTe}$ infrared sensor materials. In 1976-1977, he spent a sabbatical year at the Clarendon Laboratory, Oxford University, where he participated in research on quantum transport, infrared spectroscopy, and electrooptic measurements in semiconductors. In 1978, he joined the $\mathrm{Na}$ tional Bureau of Standards to develop microelectronic test structures. He designed the clean room facility and now directs the research program of the NBS Semiconductor Processing Research Laboratory. He is presently group leader of the Process Metrology Group in the Semiconductor Electronics Division. His personal research is on processed materials characterization, such as metal-semiconductor contacts, dopant density profiling, and ion-implantation activation.

Dr. Carver is a member of the AAAS, the APS, the IES, and numerous environmental organizations.

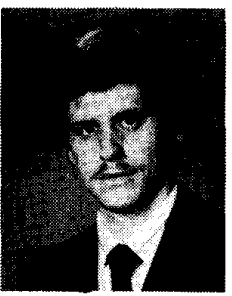

Joseph J. Kopanski (M'87) was born in Cleveland, $\mathrm{OH}$, on February 8,1960 . He received the B.S. degree in applied physics and the M.S. degree in electrical engineering and applied physics from Case Western Reserve University, Cleveland, in 1982 and 1985 , respectively. His thesis topic was the development of processing and characterization technology for silicon carbide semiconductor devices.

He joined the National Bureau of Standards, Center for Electronics and Electrical Engineering, Semiconductor Electronics Division, as an Electrical Engineer in 1985 , where he is associated with the NBS Semiconductor Processing Research Laboratory. His research interests are semiconductor process metrology and device characterization. At the NBS, he has been involved in the development of the measurement of metal-semiconductor contact resistance, low-temperature process metrology, and silicon carbide device development.

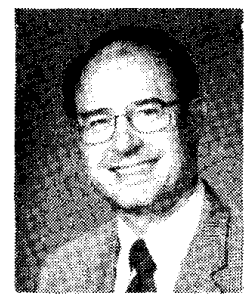

Donald B. Novotny (M'80) received the B.S. degree in chemistry in 1959 from the University of Iowa, Iowa City, and the Ph.D. degree in physical chemistry in 1964 from Iowa State University, Ames.

From 1964 to 1966, he served as Senior Research Chemist at the Monsanto Research Corp. where he worked on phase stability problems of fast breeder reactor fuels. In 1966-1967, he served at the Massachusetts Institute of Technology, Department of Chemistry, where he investigated ultrasonic properties and phase transitions of inorganic materials. Since 1967, 
he has been with the National Bureau of Standards. His work at NBS has included high-vacuum technology, attenuated total reflectance spectroscopy, and photolithography. He carried out independent original research on photoresist characterization, particularly photoresist sensitometry. $\mathrm{He}$ has been responsible also for developing and conducting programs to establish standards for lithographic linewidth calibration, for automated photomask inspection, and for photoresist and photomask materials. In 1980 , he became Manager of the Semiconductor Processing Research Laboratory. Recently, he played a leading role in the design of a new clean room facility and in the development of an expanded processing research program.

Dr. Novotny is a member of the APS and the ECS

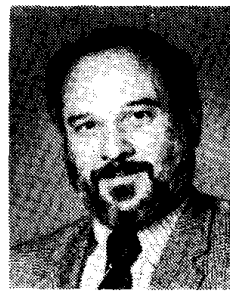

Richard A. Forman received the B.S. degree in physics from the Polytechnic Institute of Brooklyn, Brooklyn, NY, in 1959 and the Ph.D. degree in physics from the University of Maryland, College Park, in 1965.

He joined the National Bureau of Standards in 1959, working part time until he completed his doctoral studies, and he has been there continuously. He has worked in the fields of transport, magnetic resonance, optical spectroscopy, highpressure physics, and X-ray scattering as applied to materials characterization since he joined NBS. He is currently working on photoluminescence, $\mathrm{X}$-ray topography, and EXAFS as applied to semiconductor characterization.

Dr. Forman is a member of the American Physical Society and the Japanese Society for Applied Physics. 